\title{
Le mythe du régime crétois
}

Sophie Coavoux

\section{(2) OpenEdition}

Journals

Édition électronique

URL : http://journals.openedition.org/transtexts/604

DOI : $10.4000 /$ transtexts.604

ISSN : 2105-2549

Éditeur

Gregory B. Lee

Référence électronique

Sophie Coavoux, "Le mythe du régime crétois », Transtext(e)s Transcultures 跨文本跨文化 [En ligne]

10 | 2015, mis en ligne le 21 juin 2016, consulté le 21 décembre 2020. URL : http://

journals.openedition.org/transtexts/604; DOI : https://doi.org/10.4000/transtexts.604

Ce document a été généré automatiquement le 21 décembre 2020.

(c) Tous droits réservés 


\section{Le mythe du régime crétois}

\section{Sophie Coavoux}

1 Depuis les années 1990, la diète méditerranéenne connaît un succès international et s'est vue classée en 2010 sur la Liste représentative du patrimoine culturel immatériel de l'humanité de l'UNESCO. Dans le champ scientifique, essentiellement en médecine, on relève une pléthore de références sur le sujet, qui démontrent les bienfaits sanitaires de ce modèle alimentaire, notamment dans la prévention des maladies cardio-vasculaires. De multiples indicateurs démontrent la popularité désormais acquise, même auprès du grand public, de cette diète méditerranéenne souvent présentée comme " miraculeuse ", et de nombreux centres de recherche et autres fondations se consacrent à l'étude et à la promotion de cette dernière. ${ }^{1}$ De quoi s'agit-il exactement? Qu'est-ce que la « diète méditerranéenne » ? Les multiples définitions que l'on relève dans l'abondante littérature sur le sujet rendent compte de perceptions différentes, parfois contradictoires, selon les champs disciplinaires. ${ }^{2}$ À titre d'exemple, la définition donnée par l'UNESCO, très large, ne permet pas d'identifier précisément ce qu'est la «diète méditerranéenne ». ${ }^{3}$ À ce problème définitoire vient s'ajouter une confusion terminologique: on utilise en effet pêle-mêle les expressions "diète méditerranéenne ", "régime méditerranéen ", " alimentation méditerranéenne ", "cuisine méditerranéenne " ou "régime crétois ". Pour ce qui est du "régime crétois ", qui nous intéresse plus particulièrement ici, il fut «découvert » puis promu dès les années 1950 par Ancel Keys qui a fait des pratiques alimentaires de la Crète des années 1950-1960 le modèle originaire du "régime méditerranéen ", devenu ensuite terme générique avec lequel il est souvent confondu. ${ }^{4}$

On prête à ce régime toutes les vertus, à en juger par les titres des ouvrages consacrés à la question. Outre les vertus sanitaires que de nombreuses études médicales ont mises en avant depuis les années 1950, le régime crétois est présenté comme savoureux, authentique, millénaire, gage de longévité, écologique, facteur de sociabilité, marqueur identitaire fort. ${ }^{5}$ Dans ce contexte, difficile de ne pas trouver suspects l'anoblissement et la reconnaissance internationale de ce mode d'alimentation qui était à l'origine un exemple type de cucina povera. D'autant plus que, selon une étude menée par l'International Obesity Task Force publiée en 2005 et relayée par la Commission européenne, les Crétois détiennent aujourd'hui un triste record, celui de l'obésité 
infantile : en Crète, plus de $30 \%$ des enfants âgés de 7 à 11 ans et près de $35 \%$ des $13-17$ ans souffrent d'obésité, soit le taux le plus élevé d'Europe. ${ }^{6}$ Ce hiatus entre un modèle alimentaire présenté comme « idéal » et la réalité des pratiques alimentaires révélées par les enquêtes sanitaires, les multiples contradictions et approximations qui apparaissent autour du sujet, laissent dubitatif et invitent à l'incrédulité.

Quoi qu'il en soit, le régime crétois pose question. S'agit-il d'un idéal médical, d'un concept marketing, d'un outil idéologique ou d'une pratique alimentaire avérée? Nous nous proposons d'analyser, dans une perspective transdisciplinaire, la construction de ce concept (que n'ont pas inventé les Crétois), via différents facteurs (historiques, socioculturels, politiques et économiques), et d'esquisser les principaux enjeux du "régime crétois », en termes de pratiques comme sur le plan de l'imaginaire.

4 Il convient pour commencer de retracer les origines (américaines) de ce concept qui semble être devenu un véritable mythe. En 1948, dans le contexte de l'après-guerre, à la demande du gouvernement grec, la fondation Rockefeller confie à Leland Allbaugh une vaste étude épidémiologique visant à l'amélioration des conditions de vie des Crétois, et dont l'un des faisceaux de recherche concerne leurs habitudes alimentaires. Les résultats de l'étude, publiés en 1953, révèlent que l'alimentation des Crétois se caractérise par une consommation importante de plantes (plantes sauvages, herbes, fruits et légumes), inversement proportionnelle à leur consommation de viande et de produits laitiers, et par beaucoup d'huile d'olive vierge. ${ }^{7}$ Ces résultats, révélateurs, selon Allbaugh, d'un bon équilibre alimentaire (comparé notamment au modèle américain de l'époque) ne sont pas directement corrélés à des questions de santé. Il est intéressant de noter que cette première étude laisse apparaître que, de l'avis des principaux intéressés, ce modèle alimentaire n'est pas satisfaisant: seul un Crétois sur six, parmi l'échantillon de l'enquête, s'en dit satisfait, $72 \%$ souhaitent manger davantage de viande, et certains se plaignent même d'avoir continuellement faim. ${ }^{8}$ Le mode d'alimentation des Crétois des années 1950, vanté pour sa sobriété, était donc davantage le résultat d'une indigence subie, d'une frugalité de nécessité, plutôt que d'un choix délibéré.

La dimension sanitaire du régime crétois et la naissance du concept même émergent avec le physiologiste américain Ancel Keys, de l'Université du Minnesota. Il entame en 1947 une étude épidémiologique pour mesurer le rapport entre régime alimentaire et santé. D'abord limitée aux États-Unis, l'étude s'étend ensuite à six autres pays pour devenir la "Seven Countries Study ", incluant, outre les États-Unis, la Finlande, les Pays-Bas, l'Italie, la Yougoslavie, le Japon et la Grèce. Si la Grèce fait partie de l'étude, c'est que l'équipe du professeur Keys comptait un jeune chercheur grec, Christos Aravanis. Ayant étudié la médecine en Grèce, avant de se rendre aux États-Unis, il n'avait, selon son témoignage, jamais rencontré d'infarctus dans son pays d'origine, et se disait très surpris face à la fréquence de la maladie chez les Américains. C'est Aravanis qui a choisi les deux territoires grecs qui seraient soumis à l'étude : la Crète puis Corfou. Les conclusions générales de l'étude des sept pays sont publiées en $1980 .{ }^{9}$ Entre autres choses, l'étude démontre clairement que l'échantillon crétois était de loin le moins concerné par les maladies cardiovasculaires. Il est intéressant de signaler en outre que dès 1959, Keys et son épouse avaient déjà publié un livre de recettes, Eat well, stay well (New York, Doubleday \& Company), réédité en 1975, sous le titre How to eat well and stay well : the mediterranean way (New York, Doubleday \& Company). Dans cet ouvrage, le couple Keys suggère qu'il existe un lien étroit entre alimentation et santé : le régime méditerranéen jouerait un rôle dans la prévention des maladies coronariennes et de certains types de 
cancer, et se trouverait par conséquent associé à une espérance de vie optimale. De surcroît, les Keys soulignent les qualités gustatives de cette alimentation décrite en outre comme facteur de sociabilité ( « délices partagés »). Par ailleurs, la diète méditerranéenne y est décrite dans un registre qui tient plus de l'idéalisation romantique que de la description scientifique. Le couple Keys met littéralement en scène la naissance du concept dont la genèse (ou la cosmogonie) coïncide avec sa découverte de la Méditerranée (en Italie). Mêlant subjectivité et émotions personnelles, il échafaude ainsi une vision fantasmatique de la Méditerranée :

6 "Snowflakes were beginning to fall as we left Strasbourg on the fourth of February. All the way to Switzerland we drove in a snow-storm. The next morning Lausanne and the road up the valley was so deep in now that it was touch and go getting to Brig to load the car and ourselves on the train that goes through the twelve-mile tunnel to Italy. On the Italian side the air was mild, flowers were gay, birds were singing, and we basked at an outdoor table drinking our first espresso coffee at Domodossola. We felt warm all over, not only from the strong sun but also from a sense of the warmth of the people, a feeling we were later to experience in all of what we now call 'our' Mediterranean, that great stretch of land from the Strait of Gibraltar to where Europe ends at the cradle of European culture".

7 Quoi qu'il en soit, le concept de régime méditerranéen (Mediterranean Diet) était né, issu du régime crétois. Réifié, il se trouve, dès sa création, simultanément sacralisé par la science et mythifié, vecteur d'une vision imaginaire de la Méditerranée, incarnation d'une altérité idéale.

8 Dans la continuité des travaux d'Ancel Keys, d'autres études se succèdent ensuite (notamment une enquête menée par EURATOM de 1963-1965, une autre par l'OMS entre 1961 et 1990), venant confirmer l'intérêt sanitaire du régime méditerranéen, pauvre en cholestérol. ${ }^{10}$ En 1995 , des chercheurs américains, italiens et grecs élaborent la Pyramide du régime méditerranéen qui propose un modèle d'alimentation sain, faisant du régime méditerranéen une quasi injonction. ${ }^{11}$ Enfin, la dernière étude en date, publiée dans le New England Journal of Medicine le 25 février 2013, vient confirmer que le régime méditerranéen serait le " gold standard » en termes de prévention cardio-vasculaire. ${ }^{12}$

Soulignons par parenthèse que la conséquence directe de cette reconnaissance scientifique est la parution, partout dans le monde, à partir des années 1990, de livres de recettes « méditerranéennes » dans la lignée de l'ouvrage fondateur des Keys.

Pour ce qui est du champ scientifique, parmi les diverses études recensées, on peut en retenir une en particulier, en ce qu'elle me semble très significative pour notre sujet, puisqu'elle intronise le « miracle crétois ». Il s'agit de la Lyon Diet Heart Study - menée à la fin des années 1980. Le professeur Serge Renaud, de l'hôpital cardiologique de Lyon et directeur de recherches à l'INSERM, qui travaillait non sur le rôle du cholestérol mais sur celui des plaquettes sanguines sur la mortalité, a l'idée d'une nouvelle étude qu'il mène en collaboration avec le professeur Michel de Lorgeril. Un échantillon de 605 personnes de moins de 70 ans (dont 70\% d'hommes), est tiré au sort parmi des sujets ayant tous eu récemment un infarctus. Deux groupes sont constitués: le premier suit un régime classique préconisé pour de telles pathologies; le second doit suivre scrupuleusement un mode d'alimentation méditerranéen traditionnel, proche de celui des Crétois des années 1950. Les résultats de l'étude sont publiés dans le Lancet en juin $1994^{13}$ et sont très clairs : la mortalité cardiaque et les récidives des infarctus ont été réduites de $70 \%$ dans le groupe astreint au « régime crétois », par rapport à l'autre groupe. Et cette protection du régime 
crétois n'est pas due à une baisse du cholestérol mais à l'acide alpha-linoléique, très présent dans l'alimentation crétoise (notamment dans les escargots consommés en grande quantité). Mais l'examen de la méthodologie employée dans le cadre de cette étude apporte un bémol au succès des conclusions publiées. En effet, le « régime crétois » de la Lyon Diet Heart Study n'a finalement pas grand-chose à voir avec celui des paysans crétois des années 1950. L'huile d'olive vierge y est remplacée par de la margarine à base de colza, et les fruits et légumes crétois (pour certains variétés endogènes) par des produits de la région lyonnaise et seuls des apports nutritifs similaires servent à la référence crétoise, l'étude laissant de côté d'autres facteurs fondamentaux, liés au goût ou au mode de vie. En effet, l'étude de Lyon est appliquée en milieu hospitalier... on est bien loin du mode de vie et de l'activité physique du paysan crétois des années 1950-1960. Malgré les aspects contestables de la méthodologie et par conséquent, des résultats de l'étude, la Lyon Diet Heart Study a contribué à renforcer le mythe du régime méditerranéen, ou, pour être précis, du régime crétois. ${ }^{14} \mathrm{En}$ outre, la seule mention du régime crétois (seul ou surqualifié de divers termes tels "magique ", "authentique ", « incroyable ») dans un titre d'ouvrage semble offrir la garantie d'un succès de librairie, à en juger par la pléthore de livres sur le marché qui mettent en avant les bienfaits du régime crétois - notamment celui du professeur Serge Renaud (Le régime crétois, incroyable protecteur de notre santé, Odile Jacob, 1995).

Ce court rappel historique montre que le régime crétois/régime méditerranéen est bien distinct du mode d'alimentation des Crétois des années 1950 qui se trouvait étroitement corrélé à leur mode de vie. ${ }^{15}$ En effet, selon le recensement du Service national des statistiques ( $\mathrm{Y} \mathrm{YE}$ ), en 1961, la population de l'île était encore largement installée à la campagne (58\%), l'exode rural n'ayant commencé qu'au début des années 1960. L'économie y était essentiellement basée sur l'agriculture, et les paysans crétois vivaient en quasi autarcie (production de céréales, de légumes, élevage, cueillette des plantes; même le sel était directement ramassé sur les rochers des rivages, bien que monopole d'État). À cette époque, l'agriculture n'est pas mécanisée et l'île ne bénéficie pas de réseau routier : l'activité physique est donc intense et inévitable. ${ }^{16}$ À titre d'exemple, un Crétois marchait en moyenne à cette époque $13 \mathrm{~km}$ par jour et effectuait tous les travaux de la terre à la main. Par ailleurs, à l'époque de l'Étude des sept pays, la Grèce sort de dix ans de guerre (deuxième Guerre mondiale, occupation, guerre civile) et l'indigence se manifeste clairement dans une alimentation très pauvre. Enfin, il faut souligner un dernier facteur déterminant: celui de l'observation scrupuleuse du jeûne imposé par la religion. Six semaines avant Pâques, deux semaines avant le 15 août, 40 jours avant Noël, on ne consomme pas de produit d'origine animale (à l'exception de crustacés et de poisson certains jours), et les plus pieux jeûnent également, l'année durant, les mercredis et vendredis. L'ensemble de ces facteurs ne sont pas pris en compte dans les études qui ont façonné le concept de « régime crétois ». Par ailleurs, il apparait que ce concept a été créé au moment même où les modes d'alimentation auxquels il se référait, comme les modes de vie, étaient en train d'évoluer. L'agriculture se modernise au milieu des années 1960, et se trouve par ailleurs, sur le plan économique, très largement dépassée par le secteur touristique, dès les années 1970. L'émergence du tourisme de masse accélère très rapidement les mutations profondes des modes de vie, dont l'alimentation, en Crète comme partout en Grèce. Aujourd'hui, les Crétois ne sont épargnés ni par la mondialisation des pratiques alimentaires ni par l'industrie agroalimentaire. Leur alimentation s'est considérablement enrichie, en termes d'apports nutritionnels, et leur mode de vie a considérablement changé: moins d'exercice physique, importante 
augmentation de consommation de viande, de produits laitiers, de graisses saturées, de sucre (avec notamment la consommation de sodas), baisse significative de la consommation de fruits et légumes etc. ${ }^{17} \mathrm{La}$ consommation de viande rouge en Grèce est aujourd'hui supérieure à celle des États-Unis (100 kg par an et par habitant contre $80 \mathrm{~kg}$ aux États-Unis). ${ }^{18} \mathrm{Et}$ l'image quasi ascétique véhiculée par le concept oblitère une autre réalité : les Crétois sont de bons vivants et nombre de spécialités crétoises riment avec excès (citons par exemple, la staka, préparation à base de babeurre, l'eau de vie, consommé en grande quantité, etc.). Le surpoids a nettement progressé (notamment l'obésité, nous l'avons dit, chez les enfants et les adolescents), comme le taux de cholestérol par habitant, et les maladies cardiovasculaires ont augmenté de 117\% entre 1971 et $2001 .^{19}$

12 Ainsi, s'il ne correspond pas à des pratiques alimentaires avérées (qu'il s'agisse des Crétois des années 1950 ou de nos contemporains), et s'il n'est pas suffisamment corrélé à d'autres facteurs liés au mode de vie, le «régime crétois " apparait bien comme un concept construit, présenté comme un idéal alimentaire. Malgré tout, cette distinction est bien souvent gommée, partiellement ou totalement, dans la diffusion de ce modèle auprès du grand public qui repose sur une rhétorique relevant davantage de la mythologie que de la science.

Au-delà des arguments sanitaires, avérés ou idéalisés, et de la dimension mercantile évidente qui en découle, le régime crétois véhicule, sur le plan de l'imaginaire, bien des poncifs qui font partie intégrante de sa construction mythologique et varient selon les perspectives grecques ou non grecques. Commençons par le point de vue grec qui adjoint à l'argument sanitaire l'argument identitaire. Que l'on observe la transmission du concept dans les livres de cuisine ou via les organismes de promotion touristique, le régime crétois y est le plus souvent synonyme d'authenticité (au sens de pureté). Cette idée est directement issue du dogme de la continuité, support de l'idéologie nationale, héritage de l'historiographie romantique nationaliste. Par exemple, dans un fascicule de cuisine publié en 2003 par le Comité de promotion touristique de la Préfecture de La Canée, on lit à propos du régime crétois :

Très vite, il est apparu qu'il s'agissait là d'une fort longue histoire et d'une expérience vieille de plusieurs millénaires, remontant sans doute dès avant l'époque néolithique, bien qu'on ne dispose que de présomptions sérieuses, à défaut de preuves absolues, sur le régime des Crétois voilà quelque 5000 ans [...] d'après les découvertes provenant des fouilles archéologiques, il semble bien que les anciens Crétois aient consommé pratiquement les mêmes produits que leurs actuels descendants. [...] Les conquérants passaient, mais l'âme, la religion, la langue et la cuisine des Crétois demeuraient identiques. De cette continuité naquit une tradition qui se révèle aujourd'hui fort précieuse. $^{20}$

15 La référence, vague, à l'archéologie, quoique prudente, n'en est pas moins fallacieuse et sert avant tout à étayer le dogme de la continuité. Par exemple, pour une part importante, les légumes consommés par les Crétois ont été importés, notamment du Nouveau Monde (tomates, pommes de terre). En dépit de ces contre-vérités, l'argument de la continuité est relayé par certains chercheurs. Dans un article consultable sur Revues.org, on lit par exemple : «Rappelons qu'en Crète la civilisation minoenne atteignit son apogée au $16^{\mathrm{e}}$ siècle avant notre ère. La simplicité et le raffinement qui la caractérisaient se retrouvent aujourd'hui encore dans sa cuisine. ». 

1990 en Grèce, on retrouve très fréquemment les mêmes stratégies rhétoriques qui présentent le régime crétois à travers le prisme de la continuité culturelle, et en font un vecteur identitaire (local ou national, selon les perspectives). ${ }^{21}$ Pour certains auteurs, l'argument de la continuité vient renforcer l'idée de la pureté culturelle grecque ou crétoise, et dénient l'existence d'échanges culinaires avec des populations non-grecques, en référence, notamment, aux quatre siècles d'occupation ottomane. L'image du Crétois, irrédentiste assoiffé de liberté, et imperméable à toute altération culturelle (même culinaire), devient ainsi l'instrument d'un discours helléno-centriste ${ }^{22}$ et les pratiques alimentaires deviennent facteur de résistance aux éléments étrangers. ${ }^{23}$ s'appuie sur une authenticité fantasmatique. Le Crétois, incarnation d'une Méditerranée idéale, symbolise l'alliance du bien manger et du bien vivre. Nombre de discours le représentent via une vision figée dans le temps, ethnographique, exotique, voire primitiviste, avatar du mythe du Bon Sauvage : «les femmes vont tous les jours cueillir des plantes ", «les Crétois ont bon cœur ", "Les Crétois conservent dans de grandes jarres leur précieuse huile d'olive ", "La Crète est une terre de pêcheurs et de bergers, simples et chaleureux » (tous ces exemples sont tirés de la presse française). Ailleurs, la mythologie qui entoure le régime crétois rend compte d'une vision syncrétique du monde méditerranéen. La désignation par un terme unique, "régime méditerranéen", notamment par l'UNESCO, construit alors un modèle alimentaire à l'identité supposée forte qui ne rend pas compte des particularités locales, alors même qu'elle semble vouloir les promouvoir et les préserver face à la mondialisation et aux affres de la modernité.

Concept construit, mythifié, le régime crétois ne correspond donc pas au mode d'alimentation des Crétois. S'il est présenté par les nutritionnistes et les épidémiologistes comme une panacée, ou comme un «miracle », cela se vérifie au moins sur un point. Le concept, bon sous tous rapports, est en effet opérationnel sur bien des plans : santé, environnement, identité, mais aussi commerce. En effet, le régime crétois est aussi bon pour les finances et ses vertus roboratives profitent tant aux éditeurs et auteurs d'ouvrages de nutrition ou de cuisine, qu'aux acteurs du tourisme ou aux laboratoires pharmaceutiques qui proposent des produits dérivés. Citons, par exemple, les préparations à boire commercialisées par un laboratoire français, produit présenté comme « un complément alimentaire à base d'extraits spécifiques de l'alimentation des Crétois ». Ou encore, une boisson (dont le nom fait directement référence au mythe de l'Atlantide) qui prétend avoir mis le régime crétois en bouteille et invite le consommateur à «entrer dans la légende » de cette "île extraordinaire », lui promettant d'accéder au « miracle de longévité crétoise » qui est « bel et bien une réalité incontestable ».

En dernière analyse, l'exemple de ce mythe du régime crétois nous renseigne et nous interroge sur notre rapport à l'alimentation, sur notre perception, multifactorielle, des pratiques alimentaires, nous renvoyant à ce qu'un historien appelle notre besoin de "philological food $"^{24}$. En tout cas, il semble bien que les seuls à ne pas avoir entendu parler du régime crétois soient les Crétois eux-mêmes, du moins les derniers à suivre peu ou prou le mode d'alimentation traditionnel, en milieu rural..$^{25}$ 


\section{NOTES}

1. Citons par exemple l'IEAMED (Institut Européen de l'Alimentation Méditerranéenne), la FDMED (Fundación Dieta Mediterránea), ou encore le CICM (Conservatoire international des cuisines méditerranéennes).

2. Sur cette diversité des définitions et perceptions, voir Patricia Crotty, «The Mediterranean Diet as a food guide. The problem of Culture and history", Nutrition today, vol. 33, n6, novembredécembre 1998, p. 230 : "It seems clear there is no one Mediterranean diet (MD). An ideal MD could be defined as a nutrient profile, a selection of foods, or as a cultural and historical artifact. However, any definition of an MD should recognize the factors that created it: social, cultural, historical, political, and economic.».

3. Voir le site de l'UNESCO: «La diète méditerranéenne est un ensemble de savoir-faire, connaissances, pratiques et traditions qui vont du paysage à la table, y compris les cultures, la récolte ou la moisson, la pêche, la conservation, la transformation, la préparation et, en particulier, la consommation d'aliments. La diète méditerranéenne se caractérise par un modèle nutritionnel qui est demeuré constant dans le temps et l'espace et dont les principaux ingrédients sont l'huile d'olive, les céréales, les fruits et légumes frais ou séchés, une proportion limitée de poisson, produits laitiers et viande, et de nombreux condiments et épices, le tout accompagné de vin ou d'infusions, toujours dans le respect des croyances de chaque communauté. Mais la diète ( $\mathrm{du}$ grec diaita ou mode de vie) méditerranéenne recouvre beaucoup plus que la seule nourriture. Elle favorise les contacts sociaux, les repas collectifs étant la clé de voûte des coutumes sociales et des événements festifs. Elle a donné naissance à un formidable corpus de savoirs, chants, maximes, récits et légendes. Elle s'enracine dans le respect du territoire et de la biodiversité, et assure la conservation et le développement des activités traditionnelles et de l'artisanat liés à la pêche et à l'agriculture dans les communautés méditerranéennes dont Soria en Espagne, Koroni en Grèce, Cilento en Italie et Chefchaouen au Maroc représentent des exemples. Les femmes jouent un rôle particulièrement vital dans la transmission du savoir-faire, dans la connaissance des rituels, de la gestuelle et des célébrations traditionnelles, et enfin dans la sauvegarde des techniques. ».

http://www.unesco.org/archives/multimedia/index.php?

$\mathrm{s}=$ films_details\&pg=33\&id=1680\#.U_c7ZEBpGkw (page consultée le 20 novembre 2013)

4. Sur la distinction-confusion du regime méditerranéen et du regime crétois, voir Marion Nestle, «Mediterranean Diets: historical and research overview", The American Journal of Clinical Nutrition, 61 (suppl.), American Society for Clinical Nutrition, 1995, 1317S : «Because Keys' studies found the typical dietary pattern of the Greek island of Crete in the 1950s and 1960s to be associated with especially good health, this pattern has come to be viewed as the model Mediterranean diet. Because olive oil was a principal source of fat in the Cretan diet, the model has been extended to include diets consumed in olive-producing Mediterranean regions. In this manner, the generic term "Mediterranean diet" is used in practice to refer to dietary patterns similar to those of Crete in the early1960s and other regions in the Mediterranean where olive oil is a major fat source." $"$

5. Une étude publiée en 2008 préconise même le régime crétois contre le rhume des foins, cf. Alexandra Eschkenazi, «Contre le rhume des foins, essayez le régime crétois », Le Parisien, 25 mars 2008.

http://www.leparisien.fr/societe/contre-le-rhume-des-foins-tentez-le-regime-

cretois-25-03-2008-3297100218.php\#xtref=http\%3A\%2F\%2Fwww.google.fr\%2Furl\%3Fsa\%3Dt\% 
24rct\%3Dj\%24q\%3D\%24esrc\%3Ds\%24source\%3Dweb\%24cd\%3D6\%24cad\%3Drja\%24uact\%3D8\%

24ved\%3DOCEEQFjAF\%24url\%3Dhttp\%253A\%252F\%252Fwww.leparisien.fr\%252Fsociete\%

252Fcontre-le-rhume-des-foins-tentez-le-regime-cretois-25-03-2008-3297100218.php\%24ei\%

3DU0r3U4y-EszWauvBgdgO\%24usg\%3DAFQjCNH3e_hdRzH8ptQ-MCSG4eTX9I-Nfg\%24bvm\%

3Dbv.73373277\%2Cd.d2s

6. International Obesity Task Force, EU Platform Briefing Paper, prepared in collaboration with the European Association for the Study of Obesity, March 15 2005, Brussels.

http://ec.europa.eu/health/ph_determinants/life_style/nutrition/documents/iotf_en.pdf

(page consultée le 20 novembre 2013)

7. L. G. Allbaugh, Crete: a case study of an underdeveloped area, Princeton, Princeton University Press, 1953.

8. Cf. Nestle (1995), p. 1315S.

9. Keys Ancel et alii « Seven countries. A multivariate analysis of death and coronary heart disease », Cambridge, Harvard University Press, 1980.

10. Pour la première, voir $M$. Cresta et alii «Étude des consommations alimentaires des populations de onze régions de la Communauté Européenne en vue de la détermination des niveaux de contamination radioactive ", Rapport établi au Centre d'Étude nucléaire de Fontenayaux-Roses, EURATOM, CEA « EUR $4218 \mathrm{f}$ », Bruxelles, 1969. Pour la seconde, WHO, FAO, « Food and Health indicators in Europe: nutrition and health 1961-1990", Copenhagen, World Health Organization Regional Office for Europe, 1993.

11. W. C. Willet, F. Sacks, A. Trichopoulou, G. Drescher, A. Ferro-Luzzi, E. Helsing, D. Trichopoulos, "Mediterranean diet pyramid: a cultural model for healthy eating ", American Journal of Clinic Nutrition, vol. 61, n6, juin 1995, p. 1402S-1406S.

12. R. Estruch et alii, "Primary Prevention of Cardiovascular Disease with a Mediterranean Diet », The New England Journal of Medicine, vol. 368, n¹4, April 4, 2013.

http://www.nejm.org/doi/pdf/10.1056/NEJMoa1200303

(page consultée le 22 août 2014)

13. De Lorgeril, Renault et alii, «Mediterranean alpha-linolenic acid-rich diet in the secondary prevention of coronary heart disease », The Lancet, 11 juin 1994, 343, p. 1454-59 (1994 publication de l'analyse intermédiaire, puis les résultats seront publiés en 1999).

14. Pour le professeur Serge Renaud, le régime crétois se caractérise par davantage de céréales, de légumes et de fruits, moins de viande et de poisson, l'utilisation de l'huile d'olive comme seule graisse d'ajout, la consommation des plantes sauvages, telles le pourpier, et d'escargots (dont la composition en Crète est spécifique), riches en acide alpha-linolénique.

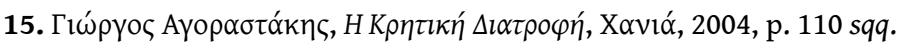

16. On se déplace à pied ou à dos d'âne. Les troupeaux n'ont pas d'enclos et les bergers suivent les troupeaux.

17. Joanna Moschandreas and Anthony Kafatos, «Food and nutrient intakes of Greek (Cretan) adults. Recent data for food-based dietary guidelines in Greece », British Journal of Nutrition, 81, Suppl. 2, 1999, S71-S76. Voir aussi Nikos Alexandratos, «The Mediterranean diet in a world context ", Public Health Nutrition, 9(1A), 2006, p. 111-117. Sur les changements des habitudes

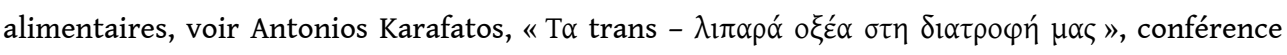
donnée le 11 mars 2014 (http://conferences.lib.auth.gr/foodchemlab/Transfat/paper/view/339) 18. Voir Karafatos, ibid.

19. D'après le Service national des statistiques ( $E \Sigma Y \mathrm{YE})$. Sur cette évolution, voir aussi Démosthènes B. Panagiotalos, Christos Pitsavos, Christina Chrysohoou, Ioannis Skoumas, Christodoulos Stefanidis, «Prevalence and Five-Year Incidence (2001-2006) of Cardiovascular Disease Risk Factors in a Greek Sample: The ATTICA Study ", Hellenic Journal of Cardiology, 50, 2009, p. 388-395.

Transtext(e)s Transcultures 跨文本跨文化, 10 | 2015 
20. L'alimentation crétoise. 24 recettes traditionnelles authentiques, Comité de promotion touristique de la Canée, La Canée, 2003.

21. Sur le sujet, voir Eric L. Ball, «Greek Food After Mousaka : Cookbooks, "Local" Culture, and the Cretan Diet », Journal of Modern Greek Studies, vol. 21, n 1 , mai 2003, John Hopkins University Press, p. 1-36.

22. Ibid. p. 13 sqq.

23. Certains ouvrages n'optent pas pour cette perspective, cf. Vassiliki Yiakoumaki, «"Local", "Ethnic", and "Rural" Food: On the Emergence of "Cultural Diversity" in Greece since its Integration in the European Union ", Journal of Modern Greek Studies, vol. 24, n², octobre 2006, p. 415-445: «At the same time new culinary manuals appear, with an apparent intention to avoid essentialist constructions of national cuisines: some contribute to a Greco-Turkish culinary encounter and "friendship" » (p. 13).

24. Zachary Nowak, «Selling the Mediterranean Diet: Marketing a Myth» (Talk given at the Echioltremare Conference, Rome, 18 June 2011).

http://www.academia.edu/1713786/Selling_the_Mediterranean_Diet_Marketing_a_Myth

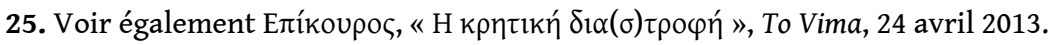

\section{RÉSUMÉS}

Souvent présentée comme «miraculeuse ", la diète méditerranéenne connaît un succès international, surtout depuis les années 1990. Mais les multiples définitions que l'on relève dans l'abondante littérature sur le sujet rendent compte de perceptions différentes, parfois contradictoires, selon les champs disciplinaires. À ce problème définitoire vient s'ajouter une confusion terminologique: on utilise en effet pêle-mêle les expressions «diète méditerranéenne », « régime méditerranéen », « alimentation méditerranéenne », « cuisine méditerranéenne » ou « régime crétois ». S'agit-il d'un idéal médical, d'un concept marketing, d'un outil idéologique ou d'une pratique alimentaire avérée? Ce texte analyse, dans une perspective transdisciplinaire, la construction de ce concept, via différents facteurs (historiques, socioculturels, politiques et économiques), et esquisse les principaux enjeux du «régime crétois ", en termes de pratiques comme sur le plan de l'imaginaire.

The Cretan Diet Myth

Since the 1990s, often described as "miraculous", the Mediterranean diet has become an international success. But the multiple definitions used in the abundant literature on the subject reflect different perceptions, sometimes contradictory, which vary among disciplines or fields of research. At this definitional problem is added a terminological confusion: diverse expressions as "Mediterranean diet", "Mediterranean food", "Mediterranean cuisine" or "Cretan diet" are jumbled together. Is it a medical ideal, a marketing concept, an ideological tool or a real feeding practice? This paper analyzes, from a transdisciplinary perspective, the construction of this concept through various factors (historical, socio-cultural, political and economic) and it outlines the main issues of the "Cretan diet" related to practices and to imaginary. 


\section{AUTEUR}

\section{SOPHIE COAVOUX}

Sophie COAVOUX est Maître de conférences de grec moderne à l'Université Jean Moulin (Lyon 3) et membre de l'IETT (Institut d'Études Transtextuelles et Transculturelles). Sa thèse de doctorat sur le poète grec C. P. Cavafy a été soutenue en 2008. Ses travaux portent sur la littérature grecque moderne et sur les questions du genre et de la diaspora. Sophie COAVOUX is Maître de conférences in Modern Greek Studies at the Jean Moulin University (Lyon 3) and a member of the IETT (Institute for Transtextual and Transcultural Studies). Her doctorate on the Greek poet C. P. Cavafy was completed in 2008. Her current research focuses on Modern Greek literature, gender and diaspora. 